\title{
Editorial
}

\section{Nonlinear Systems: Asymptotic Methods, Stability, Chaos, Control, and Optimization}

\author{
Geraldo Nunes Silva, ${ }^{1}$ Maria do Rosário de Pinho, ${ }^{2}$ \\ Marco Antonio Teixeira, ${ }^{3}$ Marcelo Messias, $^{4}$ and Elbert Macau ${ }^{5}$ \\ ${ }^{1}$ Universidade Estadual Paulista (UNESP), Instituto de Biociências, Letras e Ciências Exatas (IBILCE), \\ 15054-000 São José do Rio Preto-SP, Brazil \\ ${ }^{2}$ Faculdade de Engenharia, Universidade do Porto, Rua Dr. Roberto Frias, s/n 4200-465 Porto, Portugal \\ ${ }^{3}$ IMECC-UNICAMP, Rua Sérgio Buarque de Holanda, 651 Cidade Universitária, \\ 13083-859 Campinas, SP, Brazil \\ ${ }^{4}$ Departamento de Matemática, Estatística e Computação, FCT-UNESP, Cx. Postal 266, \\ 19060-900 Presidente Prudente-SP, Brazil \\ ${ }^{5}$ Laboratório Associado de Computação e Matemática Aplicada (LAC/CTE/INPE), \\ 12227-010 São Jósé dos Campos, SP, Brazil
}

Correspondence should be addressed to Geraldo Nunes Silva, gsilva@ibilce.unesp.br

Received 27 September 2011; Accepted 27 September 2011

Copyright (c) 2011 Geraldo Nunes Silva et al. This is an open access article distributed under the Creative Commons Attribution License, which permits unrestricted use, distribution, and reproduction in any medium, provided the original work is properly cited.

The area of nonlinear systems and control has expanded dramatically in the past fifty years. The striking developments can be credited to the increasing computing capability witnessed in the last decades which made it a lot easier to model and study real-world complex problems. This special issue puts together a set of twenty-four high-quality papers addressing a wealth of issues dealing with theoretical and/or numerical aspects of nonlinear systems stability and control design with engineering applications.

The first paper, "Numerical integration and synchronization for the 3-dimensional metriplectic Volterra system," is by Gheorghe Ivan, Mihai et al. It focuses on a metriplectic system associated to 3-dimensional Volterra model where the stability problem and numerical integration via Kahan's integrator are studied. The synchronization problem for two coupled metriplectic Volterra systems is also discussed.

The second paper is "Nonlinear dynamics of a periodically driven Duffing resonator coupled to a Van der Pol oscillator" by X. Weiet al. Here, the dynamics of a periodically driven Duffing resonator coupled elastically to a van der Pol oscillator in the case of $1: 1$ internal resonance (with weak and strong coupling) is considered. While the strong coupling leads to dominating synchronization, the weak coupling case leads to a multitude of complex behaviours. A two time scales method is proposed to obtain the frequency-amplitude modulation. The stability of the dynamic motions is also analyzed. 
Next, we have the third paper entitled "Chaos synchronization using adaptive dynamic neural network controller with variable learning rates." The authors are Chih-Hong Kao et al. The authors address the synchronization of chaotic gyros with unknown parameters and external disturbance via an adaptive dynamic neural network control (ADNNC) system. The proposed ADNNC system is composed of a neural controller and a smooth compensator.

Next we have the paper "Hybrid Taguchi-differential evolution algorithm for parameter estimation of differential equation models with application to HIV dynamics" by Wen-Hsien Ho and Agnes Lai-Fong Chan. This work centers on solving the problem of parameter estimation for a human immunodeficiency virus (HIV) dynamical model by using an improved differential evolution, called the hybrid Taguchi-differential evolution (HTDE). The HTDE approach is effectively applied to solve the problem of parameter estimation for an HIV dynamical model.

"Vibration attenuation in rotating machines using smart spring mechanism" by Aldemir Ap. Cavalini Jr. et al. is the fifth paper of this special issue. It proposes a semiactive vibration control technique dedicated to a rotating machine passing by its critical speed during the transient rotation, using a smart spring mechanism (SSM). A genetic algorithm (GA) optimization technique is used to determine the best design of the SSM parameters with respect to performance indexes associated with the control efficiency.

Next, we have "Deformation calculating of electromagnetic launcher's rail subjected to sinusoidal magnetic pressure" by Liu Wen et al.

The electromagnetic launchert's rail can be modeled as a beam on elastic foundation with simply supported beam by moving load. In this paper, Euler beam theory is applied to build a mechanical model. Also the analytical solution of the equation subjected to sinusoidal magnetic pressure is derived in detail avoiding the errors caused by using the uniform pressure to approximately replace the variable force.

We then move to "Homotopy analysis method for nonlinear dynamical system of an electrostatically actuated microcantilever" by Y. M. Chen et al. In this paper, the homotopy analysis method is employed to propose an approach for solving the nonlinear dynamical system of an electrostatically actuated microcantilever in MEMS.

In "Less conservative $\mathfrak{d}_{\infty}$ fuzzy control for discrete-time takagi-sugeno systems," Leonardo Amaral Mozelli and Reinaldo Martinez Palhares propose a new analysis and control design conditions for discrete-time fuzzy systems. By using fuzzy Lyapunov functions and introducing slack variables, less conservative conditions are obtained.

In "A note on stabilization of discrete nonlinear systems," the authors, Fengjun Tang and Rong Yuan, concentrate on the stabilization problem of discrete nonlinear systems. We construct a control Lyapunov function on discrete nonlinear systems. A new method to construct a continuous state feedback law is also presented.

Paper number 10 of our special issue is "Adaptive control of chaos in Chua's circuit" by Weiping Guo and Diantong Liu. Here, a feedback control method and an adaptive feedback control method are proposed for the ChuaŠs circuit chaos system.

Next, we have a paper entitled "Multipulse chaotic dynamics for a laminated composite piezoelectric plate" written by J. H. Zhang and W. Zhang. They investigate the global bifurcations and multipulse chaotic dynamics of a simply supported laminated composite piezoelectric rectangular thin plate under combined parametric and transverse excitations.

Paper 12 is "Interval type-2 recurrent fuzzy neural system for nonlinear systems control using stable simultaneous perturbation stochastic approximation algorithm" by Ching-Hung Lee and Feng-Yu Chang. This paper proposes a new type fuzzy neural systems, denoted IT2RFNSA (interval type-2 recurrent fuzzy neural system with asymmetric membership function), for nonlinear systems identification and control. 
In "Generalized synchronization between two complex dynamical networks with time-varying delay and nonlinear coupling," Qiuxiang Bian and Hongxing Yao investigate the generalized synchronization between two complex networks with nonlinear coupling and time-varying delay.

Paper number 14 is "New nonlinear controller for a class of chaotic systems based on adaptive backstepping fuzzy-immune control" by Kai-Shiuan Shih et al. An adaptive backstepping fuzzyimmune controller for a class of chaotic systems is proposed.

"Nonlinear mathematical modeling in pneumatic servo position applications" is a paper by Antonio Carlos Valdiero et al. It addresses a new methodology for servo pneumatic actuators mathematical modeling and selection from the dynamic behavior study in engineering applications.

Now, "The nonlinear instability modes of dished shallow shells under circular line loads" is authored by Liu Chang-Jiang et al. It investigates the nonlinear stability problem of dished shallow shells under circular line loads.

In "Digital control of a continuous stirred tank reactor," M. P. Di Ciccio et al. project a novel digital control law for continuous stirred tank reactors, based on sampled measures of temperatures and reactant concentration, as it happens in practice. The methodology of relative degree preservation under sampling is used.

Our next paper is "Dynamics of an autoparametric pendulum-like system with a nonlinear semiactive suspension" by Krzysztof Kecik and Jerzy Warminski. This paper presents vibration analysis of an autoparametric pendulum-like mechanism subjected to harmonic excitation. To improve dynamics and control motions, a new suspension composed of a semiactive magnetorheological damper and a nonlinear spring is applied.

In "A flatness-based controller for the stabilization of the inverted pendulum," Carlos Aguilar-Ibáñez et al. propose a flatness-based approach for controlling the inverted pendulum cart system, under the assumption that the pendulum state is always located on a vicinity of its unstable equilibrium point.

Next we have "A multivariable adaptive control approach for stabilization of a carttype double inverted pendulum" by I. Hassanzadeh et al. The authors consider the design and practical implementation of linear-based controllers for a cart-type double inverted pendulum (DIPC). A constitution of two linked pendulums was placed on a sliding cart, presenting a three-degree-of-freedom and single controlling input structure. The controller objective is to keep both pendulums in an up-up unstable equilibrium point.

Then, we have "Fictitious domain technique for the calculation of time-periodic solutions of scattering problem" by Ling Rao and Hongquan Chen. In this paper, the fictitious domain technique is coupled to the improved time-explicit asymptotic method for calculating timeperiodic solution of wave equation.

In "Planetary satellite orbiters: applications for the moon," Jean Paulo dos Santos Carvalho, et al. present an analytical theory with numerical simulations to study the orbital motion of lunar low-altitude artificial satellite.

The paper entitled "Solution to the linear fractional differential equation using adomian decomposition method" was written by Jin-Fa Cheng and Yu-Ming Chu. In this paper, the authors report on the analytical general solution of the linear fractional differential equations with constant coefficients by Adomian decomposition method under nonhomogeneous initial value condition, which is in the sense of the Caputo fractional derivative.

Finally, the last paper of this special issue is "An algorithm for optimally fitting a wiener model," authored by Lucas P. Beverlin et al. This work introduces a new methodology for fitting Wiener networks to datasets with a large number of variables. 


\section{Acknowledgment}

The authors would like to thank all those who have contributed to this special issue. They sincerely hope that this collection of papers may inspire many of those researchers working in this subject.

Geraldo Nunes Silva

Maria do Rosário de Pinho

Marco Antonio Teixeira

Marcelo Messias

Elbert Macau 


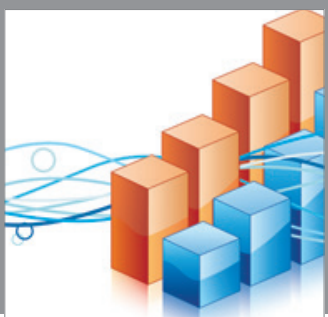

Advances in

Operations Research

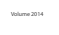

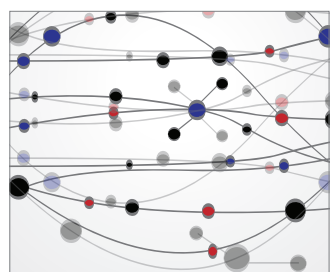

\section{The Scientific} World Journal
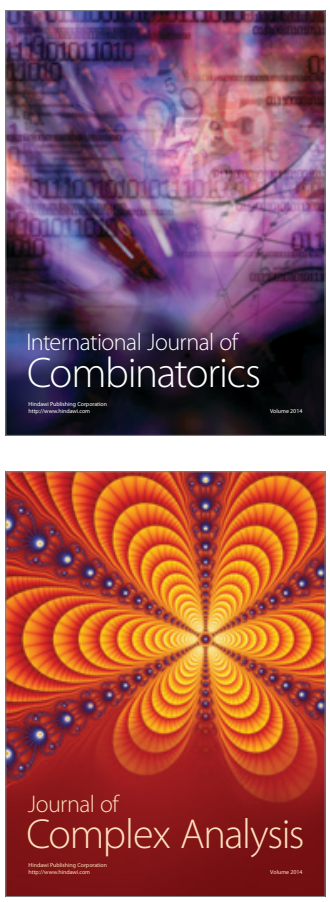

International Journal of

Mathematics and

Mathematical

Sciences
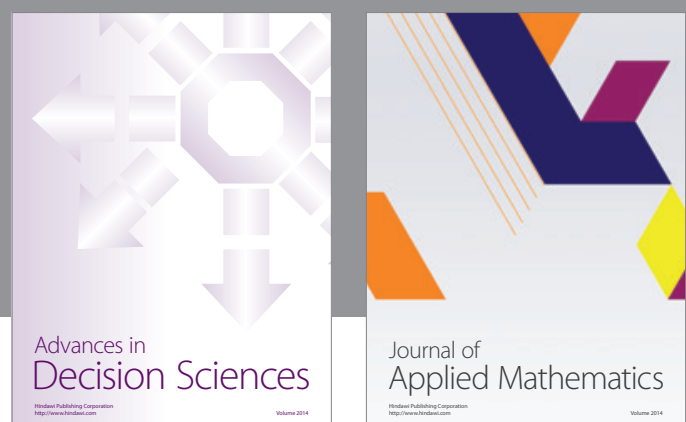

Journal of

Applied Mathematics
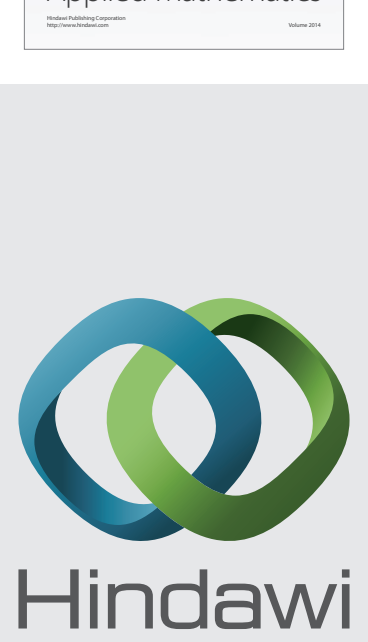

Submit your manuscripts at http://www.hindawi.com
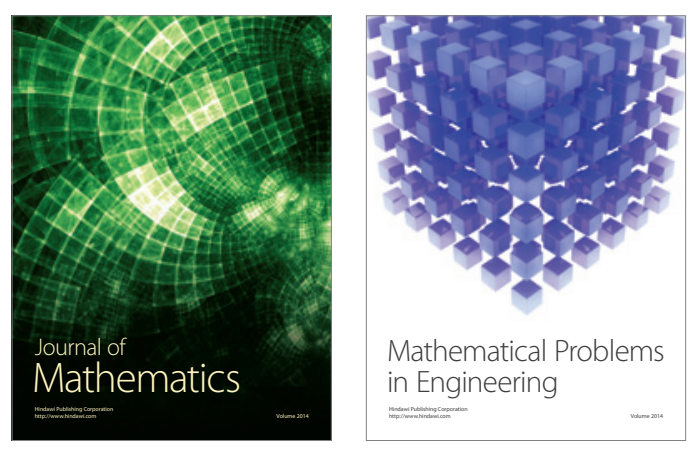

Mathematical Problems in Engineering
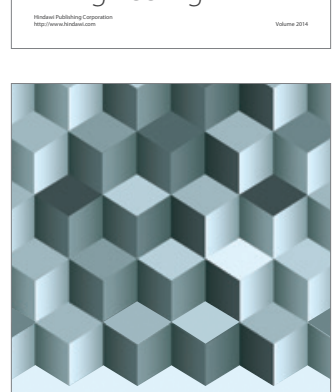

Journal of

Function Spaces
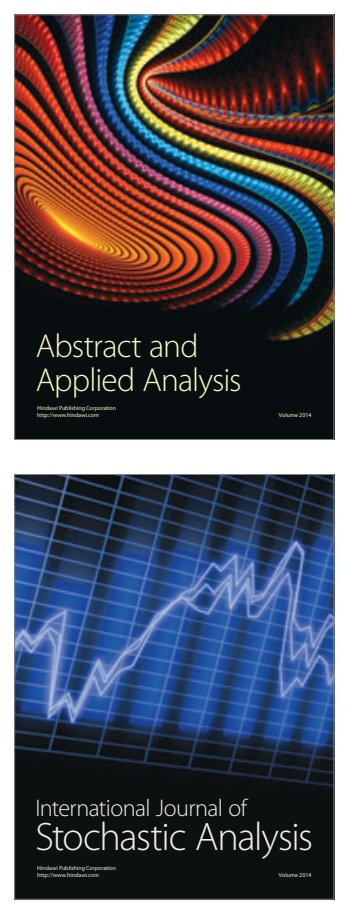

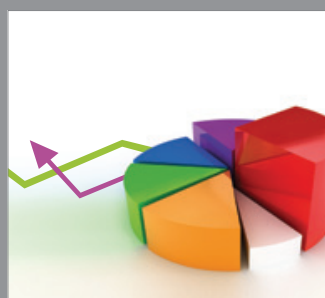

ournal of

Probability and Statistics

Promensencen
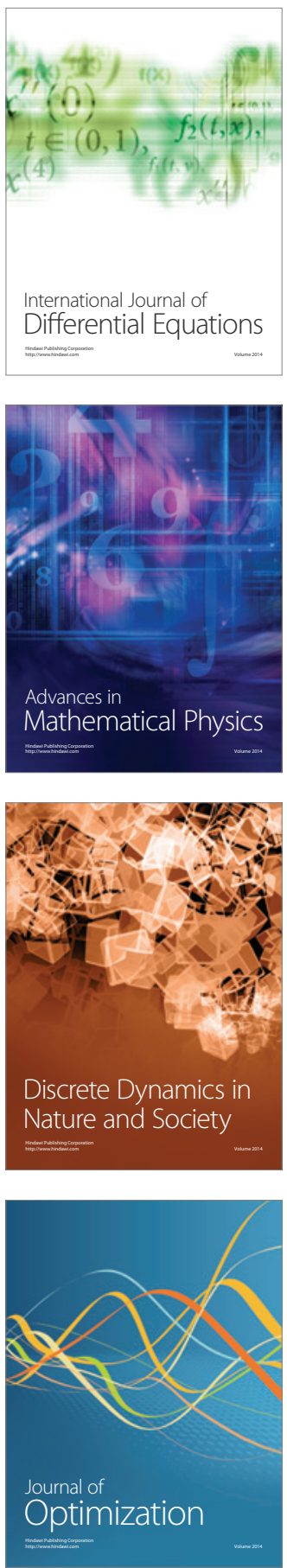